\title{
Structural diversity in binary nanoparticle superlattices
}

\author{
Elena V. Shevchenko ${ }^{1,2 \star} \uparrow$, Dmitri V. Talapin ${ }^{1 \star} \uparrow$, Nicholas A. Kotov ${ }^{3}$, Stephen O'Brien ${ }^{2} \&$ Christopher B. Murray ${ }^{1}$
}

Assembly of small building blocks such as atoms, molecules and nanoparticles into macroscopic structures-that is, 'bottom up' assembly-is a theme that runs through chemistry, biology and material science. Bacteria ${ }^{1}$, macromolecules ${ }^{2}$ and nanoparticles ${ }^{3}$ can self-assemble, generating ordered structures with a precision that challenges current lithographic techniques. The assembly of nanoparticles of two different materials into a binary nanoparticle superlattice $(\mathrm{BNSL})^{3-7}$ can provide a general and inexpensive path to a large variety of materials (metamaterials) with precisely controlled chemical composition and tight placement of the components. Maximization of the nanoparticle packing density has been proposed as the driving force for BNSL formation ${ }^{3,8,9}$, and only a few BNSL structures have been predicted to be thermodynamically stable. Recently, colloidal crystals with micrometre-scale lattice spacings have been grown from oppositely charged polymethyl methacrylate spheres $^{10,11}$. Here we demonstrate formation of more than 15 different BNSL structures, using combinations of semiconducting, metallic and magnetic nanoparticle building blocks. At least ten of these colloidal crystalline structures have not been reported previously. We demonstrate that electrical charges on sterically stabilized nanoparticles determine BNSL stoichiometry; additional contributions from entropic, van der Waals, steric and dipolar forces stabilize the variety of BNSL structures.

Face-centred-cubic (f.c.c.) ordering of monodisperse hard spheres dispersed in a liquid permits larger local free space available for each sphere compared to the unstructured phase, resulting in higher translational entropy of the spheres. When the volume fraction of hard spheres approaches $\sim 55 \%$, this ordering enhances the total entropy of the system and drives the ordering phase transition. Entropy-driven crystallization has been studied in great detail both theoretically ${ }^{12}$ and experimentally on monodisperse latex particles, whose behaviour can be approximated by hard spheres ${ }^{13,14}$. In a mixture containing spheres of two different sizes (radii $R_{\text {small }}$ and $R_{\text {large }}$ ), the packing symmetry depends on the size ratio of the small and large spheres $\left(\gamma=R_{\text {small }} / R_{\text {large }}\right)^{3,8}$. Calculations show that assembly of hard spheres into binary superlattices isostructural with $\mathrm{NaCl}, \mathrm{AlB}_{2}$ and $\mathrm{NaZn}_{13}$ can be driven by entropy alone without any specific energetic interactions between the spheres ${ }^{9,15}$. Indeed, $\mathrm{NaZn}_{13^{-}}$and $\mathrm{AlB}_{2}$-type assemblies of silica particles were found in natural Brazilian opals ${ }^{16}$ and can be grown from latex spheres ${ }^{17}$. In a certain $\gamma$ range, the packing density of these structures either exceeds or is very close to the density of the close-packed f.c.c. lattice (0.7405), while structures with lower packing densities are predicted to be unstable ${ }^{8,15}$.

Despite these predictions, we observed an amazing variety of BNSLs that self-assemble from colloidal solutions of nearly spherical nanoparticles of different materials (Fig. 1). Coherently packed domains extend up to $10 \mu \mathrm{m}$ in lateral dimensions, and can display well defined facets (Supplementary Fig. 1). In many cases, several BNSL structures form simultaneously on the same substrate, under identical experimental conditions. The same nanoparticle mixture can assemble into BNSLs with very different stoichiometry and packing symmetry. For example, 11 different BNSL structures were prepared from the same batches of $6.2 \mathrm{~nm} \mathrm{PbSe}$ and $3.0 \mathrm{~nm} \mathrm{Pd}$ nanoparticles (Supplementary Fig. 2). We also observe that, in general, BNSLs tolerate much broader $\gamma$ ranges than hard spheres: for example, $\mathrm{AlB}_{2}$-type BNSLs assembled from different combinations of $\mathrm{PbSe}, \mathrm{PbS}, \mathrm{Au}, \mathrm{Ag}, \mathrm{Pd}, \mathrm{Fe}_{2} \mathrm{O}_{3}, \mathrm{CoPt}_{3}$ and $\mathrm{Bi}$ nanoparticles in a broad $\gamma$ range (Supplementary Fig. 3). Further, we observe BNSLs that could not be identified as isostructural with specific intermetallic compounds (Supplementary Fig. 2). This observed structural diversity of BNSLs defies traditional expectations, and shows the great potential of modular self-assembly at the nanoscale.

The formation of binary structures with packing density significantly lower than the density of single-phase f.c.c. close packing (0.7405) rules out entropy as the main driving force for nanoparticle ordering. Moreover, van der Waals, steric or dipolar interparticle interactions are not sufficient to explain why these low density BNSLs form, instead of their constituents separating into single-component superlattices. Opposite electrical charges on nanoparticles could impart a specific affinity of one type of particle (for example, dodecanethiol-capped $\mathrm{Au}, \mathrm{Ag}, \mathrm{Pd}$ ) for another (typically $\mathrm{PbSe}$, $\mathrm{PbS}, \mathrm{Fe}_{2} \mathrm{O}_{3}, \mathrm{CoPt}_{3}$ and so on, capped with long chain carboxylic acids). If nanoparticles are oppositely charged, the Coulomb potential would stabilize the BNSL while destabilizing the singlecomponent superlattices. The electrical charges might be present on sterically stabilized nanoparticles even in non-polar solvents ${ }^{18-20}$.

To measure charges on the nanoparticles that form our BNSL, we studied the electrophoretic mobility of PbSe and Au nanocrystals. Laser Doppler velocimetry allows the distribution of electrophoretic mobilities within an ensemble of nanoparticles to be measured. The electrical charge $(Z$, in units of $e$ ) of a spherical particle in a low dielectric solvent in absence of electrolyte can be calculated from the electrophoretic mobility $\left(\mu_{\mathrm{e}}\right)$ where $\mu_{\mathrm{e}}=Z e /(3 \pi \eta a), \eta$ is the viscosity of the solvent and $a$ is the hydrodynamic diameter of a particle ${ }^{21}$. With $a=10 \mathrm{~nm}$, we obtain $\mu_{\mathrm{e}} \approx 0.27 \times 10^{-4} \mathrm{Zcm}^{2} \mathrm{~V}^{-1} \mathrm{~s}^{-1}$. These calculated values agree well with the peaks in the experimental mobility distribution for 7.2-nm-diameter PbSe nanocrystals in chloroform (Fig. 2a). Owing to the organic coat (oleic acid), the effective hydrodynamic radius of PbSe nanocrystals extends beyond the crystalline core by $1-2 \mathrm{~nm}$, depending on the density of surface coverage. The peaks in the mobility distribution curve indicate the 
presence of particles with charges $-e, 0, e$ and $2 e$ in a colloidal solution of monodisperse PbSe nanocrystals.

We found that the charges on PbSe nanocrystals can be altered by adding surfactant molecules like carboxylic acids and tri- $n$ alkylphosphine oxides. Addition of oleic acid increases the population of positively charged PbSe nanocrystals at the expense of the negatively charged and neutral nanocrystals. Depending on the amount of acid added, the majority of nanocrystals can be adjusted to have either one or two positive charges (Fig. $2 \mathrm{~b}$ and c). Addition of oleic acid increases the solutions' viscosity, causing the peaks to shift towards lower mobility (compare Fig. 2a-c). The addition of tri- $n$ octylphosphine oxide (TOPO) increases the population of negatively charged PbSe nanocrystals and reduces the concentration of positively charged nanocrystals (Fig. 2d). Surveys of many samples revealed that the additives reliably shifted the distribution of charge states; however, the initial proportion of particles in each charge state was dependent somewhat on sample processing. Both neutral and negatively charged nanoparticles were detected in chloroform solutions of $4.8 \mathrm{~nm}$ dodecanethiol-capped Au nanocrystals (Supplementary Fig. 5). After addition of oleic acid most Au nanoparticles become negatively charged (Fig. 2e), whereas the addition of TOPO neutralizes the Au nanoparticles (Fig. 2f). The charges on PbSe and $\mathrm{Au}$ nanoparticles could originate from deviations in nanocrystal stoichiometry and adsorption/desorption of charged capping
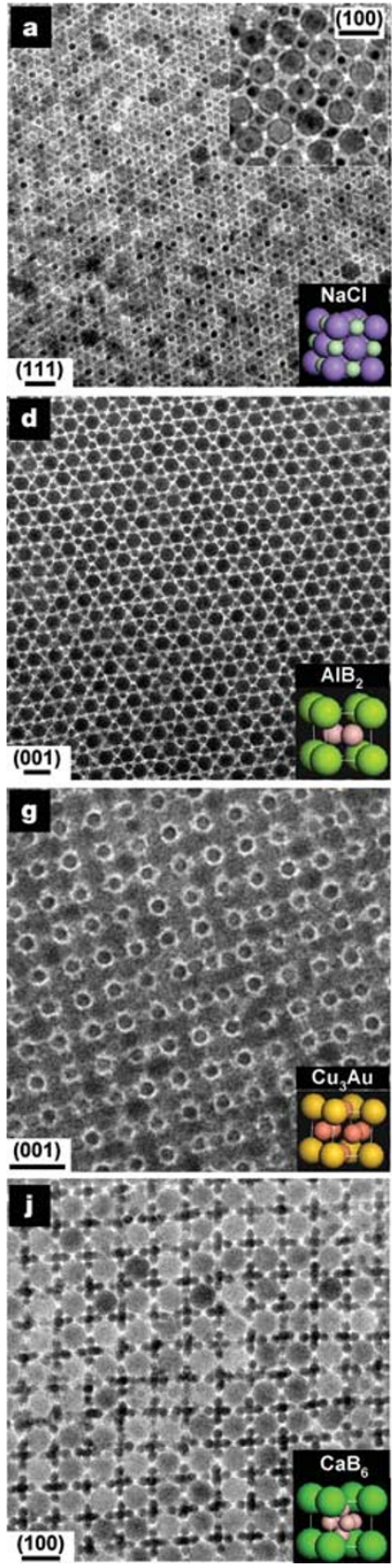

Figure 1 | TEM images of the characteristic projections of the binary superlattices, self-assembled from different nanoparticles, and modelled unit cells of the corresponding three-dimensional structures. The superlattices are assembled from a, $13.4 \mathrm{~nm} \gamma-\mathrm{Fe}_{2} \mathrm{O}_{3}$ and $5.0 \mathrm{~nm} \mathrm{Au}$; b, $7.6 \mathrm{~nm} \mathrm{PbSe}$ and $5.0 \mathrm{~nm} \mathrm{Au}$; c, $6.2 \mathrm{~nm}$ PbSe and $3.0 \mathrm{~nm}$ Pd; d, $6.7 \mathrm{~nm} \mathrm{PbS}$ and $3.0 \mathrm{~nm}$ Pd; e, $6.2 \mathrm{~nm}$ PbSe and $3.0 \mathrm{~nm}$ Pd; f, $5.8 \mathrm{~nm}$ PbSe and $3.0 \mathrm{~nm} \mathrm{Pd}$;
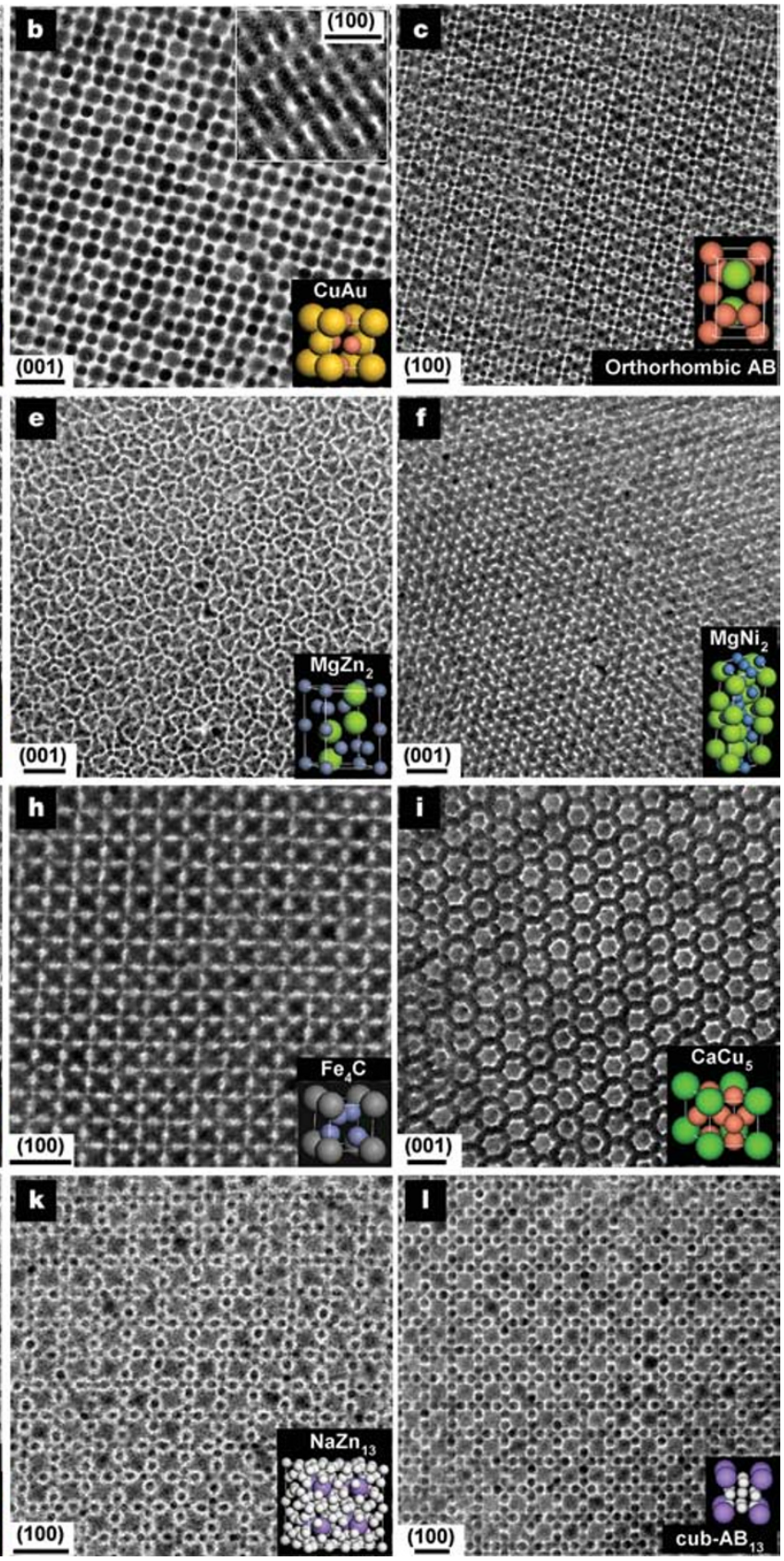

g, 7.2 nm PbSe and 4.2 nm Ag; h, $6.2 \mathrm{~nm}$ PbSe and $3.0 \mathrm{~nm}$ Pd; i, $7.2 \mathrm{~nm}$ PbSe and $5.0 \mathrm{~nm} \mathrm{Au} ; \mathbf{j}, 5.8 \mathrm{~nm}$ PbSe and $3.0 \mathrm{~nm}$ Pd; $\mathbf{k}, 7.2 \mathrm{~nm}$ PbSe and $4.2 \mathrm{~nm} \mathrm{Ag}$; and $\mathbf{l}, 6.2 \mathrm{~nm}$ PbSe and $3.0 \mathrm{~nm}$ Pd nanoparticles. Scale bars: a-c, e, f, $\mathbf{i}-\mathbf{I}$, $20 \mathrm{~nm} ; \mathbf{d}, \mathbf{g}, \mathbf{h}, 10 \mathrm{~nm}$. The lattice projection is labelled in each panel above the scale bar. The modelled projections of the binary superlattices are shown in Supplementary Fig. 4. 
ligands. Although these additives are effective in adjusting the particle charge states, the specific interactions by which this charge tuning occurs will require further study (Supplementary Discussion 1).

In the presence of oleic acid, $\mathrm{PbSe}$ and $\mathrm{Au}$ nanoparticles are oppositely charged (Fig. 2e). The Coulomb potential between two oppositely charged nanoparticles $(Z= \pm 1)$ separated by $10 \mathrm{~nm}$ of a solvent like chloroform is comparable with $k T$ at room temperature, and solutions of mixed $\mathrm{PbSe}$ and metal nanoparticles retain stability for several weeks. The relatively small interparticle potential favours annealing of the BNSLs as they grow. For a NaCl-type BNSL with $Z_{+}=1, Z_{-}=-1$ and the nearest-neighbour distance $R_{0}=11.5 \mathrm{~nm}$ (Fig. 1a), the Coulomb binding energy per unit cell is estimated to be $U_{\text {Coul }} \approx M Z_{+} Z_{-} e^{2} /\left(4 \pi \varepsilon \varepsilon_{0} R_{0}\right) \approx-0.1 \mathrm{eV}$ (or about $-4 k T$ at the superlattice growth temperature, $50{ }^{\circ} \mathrm{C}$ ), where $M=-1.7476$ is the Madelung constant. The Coulomb binding energy is comparable to the van der Waals attractive energy expected for a NaCl-type BNSL. The energy of short-range van der Waals forces $\left(\sim 1 / R^{6}\right)$ can rival long-range Coulomb energy $(\sim 1 / R)$ only at the nanometre scale. In BNSLs, we can neglect screening of the Coulomb potential by charged species in solution because the Debye screening length $\left(\sim 10^{-4} \mathrm{~cm}\right)$ is much larger than $R_{0}$ (refs 10,11$)$. In an $\mathrm{AB}_{x}$ BNSL where $\mathrm{A}$ and $\mathrm{B}$ hold opposite charges, the Coulomb potential per $\mathrm{AB}_{x}$ 'molecule' is $U_{\text {Coul }} \approx-\alpha+\beta\left(x Z_{-}+Z_{+}\right)^{2} N^{2 / 3}$, where $\alpha$ and $\beta$ are positive constants and $N$ is the number of assembled nanoparticles (Supplementary Discussion 2).

Coulomb energy determines the stoichiometry of the growing BNSL. An extended three-dimensional BNSL can form only if the positive and negative charges compensate each other. If during growth the BNSL accumulates non-compensated charge, eventually $U_{\text {Coul }}$ changes sign from negative to positive and the growth is selflimiting. The superlattice nucleation stage should be less sensitive to the Coulomb interactions. Indeed, we observed that many small domains with different BNSL structures can simultaneously nucleate on the same substrate, but their size does not exceed $\sim 10^{2}$ nanoparticles. Only one or two structures grow to larger length scales $\left(\sim 10^{6}-10^{8}\right.$ particles). BNSLs with many particles per unit cell (for
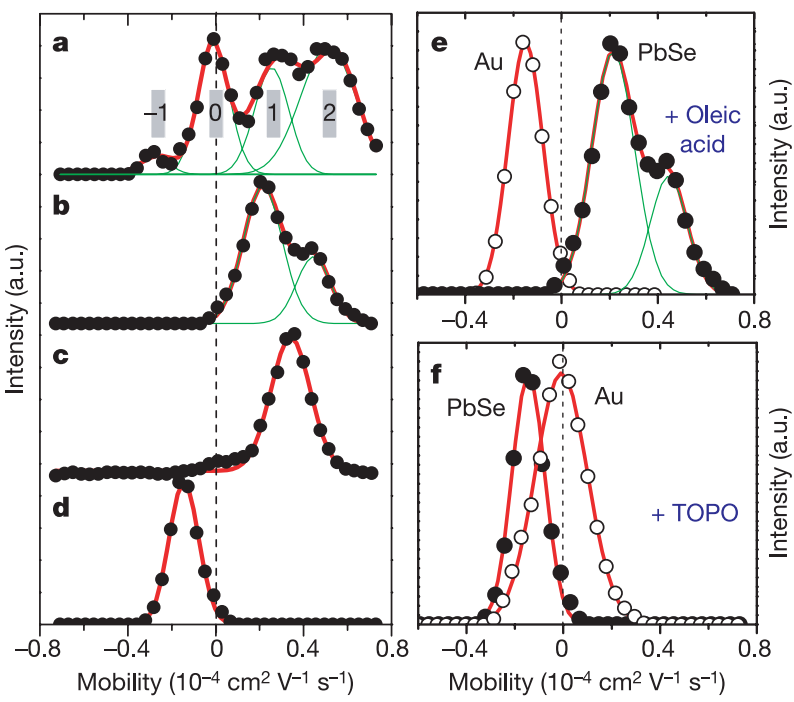

Figure 2 | Electrophoretic mobility of PbSe and Au nanocrystals in chloroform. a-d, Distribution of electrophoretic mobility for $7.2 \mathrm{~nm} \mathrm{PbSe}$ nanocrystals. a, PbSe nanocrystals washed to remove excess of capping ligands. The grey bars show mobilities predicted for nanocrystals with charges of $-1,0,1$ and 2 (in units of $e$ ). b-d, Electrophoretic mobility of PbSe nanocrystals in the presence of b, $0.02 \mathrm{M}$ oleic acid, c, $0.06 \mathrm{M}$ oleic acid and d, $0.05 \mathrm{M}$ tri- $\boldsymbol{n}$-octylphosphine oxide. e, f, Comparison of electrophoretic mobilities of $7.2 \mathrm{~nm}$ PbSe and $4.8 \mathrm{~nm}$ Au nanocrystals in the presence of e, $0.02 \mathrm{M}$ oleic acid and f, $0.05 \mathrm{M}$ tri- $\boldsymbol{n}$-octylphosphine oxide, respectively. a.u., arbitrary units. example, $\left.\mathrm{AB}_{4}, \mathrm{AB}_{5}, \mathrm{AB}_{6}, \mathrm{AB}_{13}\right)$ might form when both charged and neutral nanoparticles of type $B$ are incorporated into the structures. The presence of differently charged nanoparticles in the colloidal solutions (Fig. 2a and Supplementary Fig. 5) could also contribute to the simultaneous formation of different BNSLs. Intentional addition of a large concentration of charged species into a solution of nanoparticles might reduce the Debye screening length down to $R_{0}$, relaxing the strict rules for BNSL charge neutrality and allowing a range of new structures to be formed ${ }^{10}$.

Tuning the charge state of the nanoparticles allows us to direct the self-assembly process. Reproducible switching between different BNSL structures has been achieved by adding small amounts of carboxylic acids, TOPO or dodecylamine to colloidal solutions of $\mathrm{PbSe}\left(\mathrm{PbS}, \mathrm{Fe}_{2} \mathrm{O}_{3}\right.$, and so on) and metal (Au, $\mathrm{Ag}, \mathrm{Pd}$ ) nanocrystals. Figure 3 demonstrates how these additives direct the formation of specific BNSL structures. Combining native solutions of $6.2 \mathrm{~nm} \mathrm{PbSe}$ and $3.0 \mathrm{~nm} \mathrm{Pd}$ nanoparticles (particle concentration ratio $\sim 1: 5$ ) results in the formation of several BNSL structures with $\mathrm{MgZn}_{2}$ and cuboctahedral $\mathrm{AB}_{13}$ lattices dominating. However, the same nanoparticles assemble into orthorhombic $\mathrm{AB}$ - and $\mathrm{AlB}_{2}$-type superlattices after adding oleic acid (Fig. 3a), and into $\mathrm{NaZn}_{13^{-}}$or
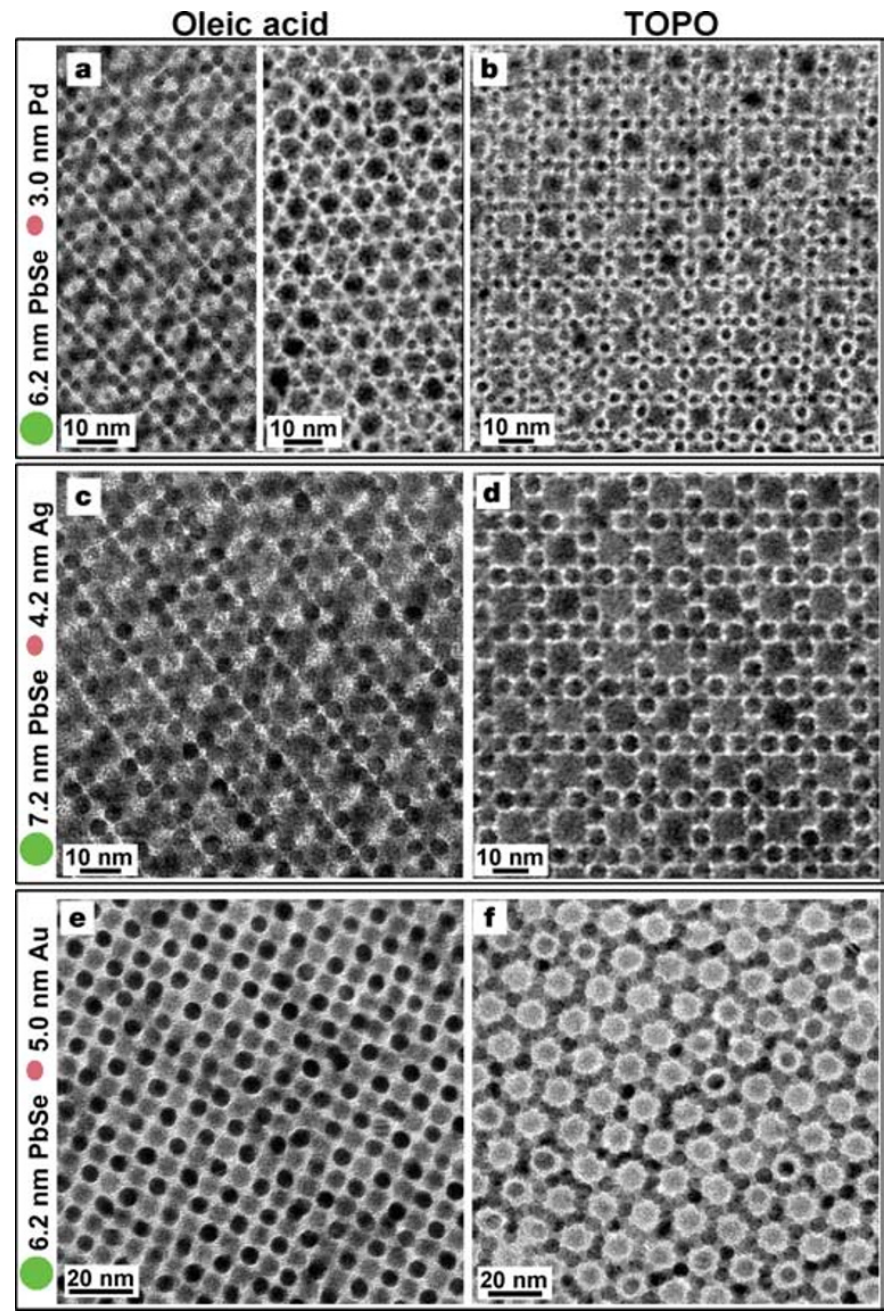

Figure 3 | TEM images of binary superlattices self-assembled in the presence of $4 \mathrm{mM}$ oleic acid (left column) and $6 \mathrm{mM}$ tri-n-octylphosphine oxide, TOPO (right column). a, $6.2 \mathrm{~nm} \mathrm{PbSe}$ and $3.0 \mathrm{~nm} \mathrm{Pd}$ nanoparticles self-assembled into orthorhombic $\mathrm{AB}$ - and $\mathrm{AlB}_{2}$-type BNSLs, and b, into $\mathrm{NaZn}_{13}$-type BNSL. c, d, $7.2 \mathrm{~nm} \mathrm{PbSe}$ and $4.2 \mathrm{~nm} \mathrm{Ag}$ nanoparticles self-assembled into orthorhombic $\mathrm{AB}$ and cuboctahedral $\mathrm{AB}_{13}$ BNSLs, respectively. e, f, $6.2 \mathrm{~nm}$ PbSe and 5.0 nm Au nanoparticles selfassembled into $\mathrm{CuAu}$-type and $\mathrm{CaCu}_{5}$-type $\mathrm{BNSLs}$, respectively. 

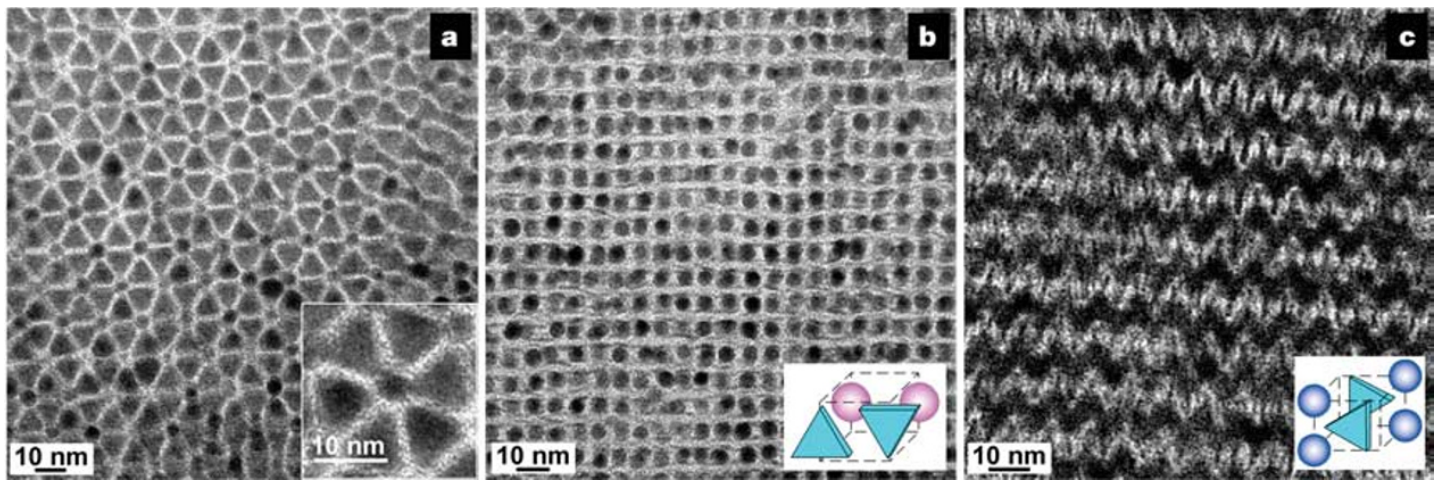

Figure 4 TEM images and proposed unit cells of binary superlattices self-assembled from triangular nanoplates and spherical nanoparticles. a, b, Self-assembled from $\mathrm{LaF}_{3}$ triangular nanoplates $(9.0 \mathrm{~nm}$ side) and $5.0 \mathrm{~nm}$ Au nanoparticles; c, self-assembled from $\mathrm{LaF}_{3}$ triangular nanoplates

cuboctahedral $\mathrm{AB}_{13}$-type BNSLs after the addition of dodecylamine or TOPO, respectively (Fig. $3 \mathrm{~b}$ ). In the $\mathrm{AB}_{13}$-type BNSL, metal particles assemble into icosahedral $\left(\mathrm{NaZn}_{13}\right)$ or cuboctahedral (cuboctahedral $\mathrm{AB}_{13}$ ) clusters, with each large PbSe particle surrounded by 24 metal spheres at the vertices of a snub cube ${ }^{7}$. In the presence of TOPO the metal nanoparticles are neutral (Fig. 2f), favouring formation of the $\mathrm{Pd}_{13}\left(\mathrm{Au}_{13}, \mathrm{Ag}_{13}\right)$ clusters. The clusters of metal nanoparticles in turn provide screening of the charges on $\mathrm{PbSe}$ nanocrystals in the $\mathrm{AB}_{13}$-type BNSL.

Surveys of many samples show that the addition of a carboxylic acid to solutions of $\mathrm{PbSe}-\mathrm{Pd}, \mathrm{PbSe}-\mathrm{Au}, \mathrm{PbSe}-\mathrm{Ag}$ and $\mathrm{PbSe}-\mathrm{Fe}_{2} \mathrm{O}_{3}$ nanoparticle mixtures results in either $\mathrm{AB}$ or $\mathrm{AB}_{2}$ superlattices (Fig. 3c, e), whereas the addition of TOPO to mixtures of the same nanoparticles favours growth of $\mathrm{AB}_{13}$ (if $\gamma<\sim 0.65$ ) or $\mathrm{AB}_{5}$ (if $\gamma>\sim 0.65$ ) BNSLs (Fig. 3d, f). Thus the space-filling principles and particle charging work in combination to determine the structure. Adjusting the relative concentrations of A and B particles can be used as an additional tool with which to control the BNSL structure. For example, in presence of TOPO, $\mathrm{AB}_{4}$ BNSLs can form when the $A: B$ ratio is $\sim 1: 1$, whereas exclusively $A B_{13}$ forms in the presence of large excess $\mathrm{B}$ particles.

In contrast to particles with amorphous or polycrystalline morphology, nanocrystals allow exploitation of the inherent crystal anisotropy to precisely engineer nanocrystal shape ${ }^{22}$. The nanocrystal shape can in turn be used as a powerful tool to engineer the structure of the self-assembled BNSLs. For example, Fig. 4 shows several BNSLs self-assembled from $\mathrm{LaF}_{3}$ triangular nanoplates and spherical $\mathrm{Au}$ or PbSe nanocrystals. In the $\mathrm{LaF}_{3}-\mathrm{Au}$ system, the $\mathrm{LaF}_{3}$ nanoplates lie flat on silicon oxide surface (Fig. 4a) and stand on edge when assembled on amorphous carbon (Fig. $4 \mathrm{~b}$ and c), demonstrating how the choice of substrate can be used to control the BNSL structure.

It is specifically at the nanoscale that the van der Waals, electrostatic, steric repulsion and the directional dipolar interactions can contribute to the interparticle potential with comparable weight ${ }^{18,23,24,25}$. These, together with the effects of particle substrate interactions and space-filling (entropic) factors, combine to determine the BNSL structure. The non-equilibrium nature of our evaporative self-assembly process adds additional complexity ${ }^{26}$. Precise control of nanoparticle size, shape and composition allows us to engineer electronic, optical and magnetic properties of nanoparticle building blocks. Assembling these nanoscale building blocks into a wide range of BNSL systems provides a powerful modular approach to the design of 'metamaterials' with programmable physical and chemical properties.

\section{METHODS}

Nanoparticle synthesis. Au, Ag and Pd nanoparticles were prepared by modifying the method of ref. 27. Metal salts were dissolved in $10 \mathrm{ml}$ of toluene with ultrasonication in the presence of dodecyldimethylammonium bromide and $6.2 \mathrm{~nm}$ PbSe nanocrystals. The insets show $\mathbf{a}$, a magnified image, and $\mathbf{b}$, c, proposed unit cells of the corresponding superlattices. The structure shown in a forms on silicon oxide surfaces, while structures shown in b and $\mathbf{c}$ form preferentially on amorphous carbon substrates.

(DDAB). For synthesis of $5.0 \mathrm{~nm} \mathrm{Au}$ and $4.2 \mathrm{~nm} \mathrm{Ag}$ nanoparticles, we used $0.034 \mathrm{~g} \mathrm{AuCl}_{3}$ and $0.025 \mathrm{~g} \mathrm{AgNO}_{3}$, respectively, and $0.0925 \mathrm{~g} \mathrm{DDAB} .3 .0 \mathrm{~nm} \mathrm{Pd}$ nanocrystals were synthesized from $0.0237 \mathrm{~g} \mathrm{PdCl}_{2}$ with $0.157 \mathrm{~g} \mathrm{DDAB}$. Forty microlitres of a $9.4 \mathrm{M}$ aqueous solution of $\mathrm{NaBH}_{4}$ were added drop-wise to the solution of metal salt with vigorous stirring. After 20 min, $0.8 \mathrm{ml} \mathrm{1-dodecanethiol}$ was added and the stirring was continued for five more minutes. The nanoparticles were precipitated by adding ethanol, and the solid redispersed in $10 \mathrm{ml}$ toluene in the presence of $0.8 \mathrm{ml} \mathrm{1-dodecanethiol} \mathrm{and} \mathrm{refluxed} \mathrm{for} 30 \mathrm{~min}$ under nitrogen. $\mathrm{Fe}_{2} \mathrm{O}_{3}$ nanocrystals were synthesized by methods adapted from ref. 28. Briefly, $11 \mathrm{~nm}$ and $13.4 \mathrm{~nm} \mathrm{Fe}_{2} \mathrm{O}_{3}$ nanocrystals were synthesized by injecting $0.2 \mathrm{ml}$ iron pentacarbonyl into $10 \mathrm{ml}$ trioctylamine in the presence of $0.65 \mathrm{~g}$ oleic acid at $270^{\circ} \mathrm{C}$ and $250^{\circ} \mathrm{C}$, respectively. After heating of the reaction mixtures at $320^{\circ} \mathrm{C}$ for $1 \mathrm{~h}$, the reaction mixture was cooled to room temperature. $0.17 \mathrm{~g}$ trimethylamine $\mathrm{N}$-oxide was added to oxidize the iron nanoparticles to $\gamma-\mathrm{Fe}_{2} \mathrm{O}_{3}$, and the reaction mixture was heated to $130^{\circ} \mathrm{C}$ for $1.5 \mathrm{~h}$ and $320^{\circ} \mathrm{C}$ for $1 \mathrm{~h}$. Details of the synthesis of $\mathrm{PbSe}, \mathrm{PbS}$ and $\mathrm{LaF}_{3}$ nanocrystals can be found in refs 7, 29 and 30, respectively. Preparation of binary superlattices. A substrate (for example, a carbon- or silicon oxide-coated transmission electron microscope (TEM) grid, a silicon nitride membrane or an alkyl-functionalized silicon chip) was placed in a glass vial containing a colloidal solution of nanoparticles. The vial was placed tilted by $60^{\circ}-70^{\circ}$ inside a low-pressure chamber. Ordered binary assemblies formed upon evaporation of the solvent. Toluene and mixtures of toluene with tetrachloroethylene or chloroform were used as solvents ( $\sim 1: 1$ by volume). The best binary assemblies (as determined by the length scale of ordering and a low occurrence of defects) were obtained by evaporating relatively concentrated colloidal solutions at $45^{\circ} \mathrm{C}$ under reduced pressure $(\sim 3.2 \mathrm{kPa})$.

Structural analysis. A Philips CM12 TEM operating at $120 \mathrm{kV}$ was used to image the structure of the assemblies. Three-dimensional descriptions of the superlattices were developed by surveying large regions of the samples, to categorize all the crystal orientations, and recording a series of two-dimensional projections down the major symmetry axes. Tilting of the samples allowed observation of additional orientations not expressed in the plan view images. To assign the observed structures to crystallographic space groups, we built three-dimensional lattice models for the 180 most common space groups using Accelrys MS Modelling 3.1 software. The TEM images were compared with simulated projections to match the symmetry of our superlattices. We also performed a comparison of experimental small-angle electron diffraction patterns taken over larger areas, and the two-dimensional Fourier transformation power spectra of real space TEM images and the fast Fourier transform power spectra of the simulated projections to assure consistency.

Electrophoretic mobility measurements. These were performed by electrophoretic light scattering using a Zetasizer Nano ZS Series (Malvern), allowing measurements in non-polar organic solvents. We used chloroform solutions with nanoparticle concentrations $\sim 5$ times higher than those used for growing binary superlattices. The concentrations of additives (oleic acid and TOPO) were similar to those used for directing BNSL self-assembly. After preparation, the colloidal solutions were left in the dark for several hours to allow the systems to equilibrate before each measurement.

Received 20 August; accepted 2 November 2005.

Shenton, W., Pum, D., Sleytr, U. \& Mann, S. Synthesis of cadmium sulphide superlattices using self-assembled bacterial S-layers. Nature 389, 585-587 (1997). 
2. Guarini, K. W., Black, C. T. \& Yeung, S. H. I. Optimization of diblock copolymer thin film self assembly. Adv. Mater. 14, 1290-1294 (2002).

3. Redl, F. X., Cho, K.-S., Murray, C. B. \& O'Brien, S. Three-dimensional binary superlattices of magnetic nanocrystals and semiconductor quantum dots. Nature 423, 968-971 (2003).

4. Kiely, C. J., Fink, J., Brust, M., Bethel, D. \& Schiffrin, D. J. Spontaneous ordering of bimodal ensembles of nanoscopic gold clusters. Nature 396, 444-446 (1998).

5. Shevchenko, E. V. et al. Colloidal synthesis and self-assembly of $\mathrm{CoPt}_{3}$ nanocrystals. J. Am. Chem. Soc. 124, 11480-11485 (2002).

6. Saunders, A. E. \& Korgel, B. A. Observation of an AB phase in bidisperse nanocrystal superlattices. ChemPhysChem 6, 61-65 (2005).

7. Shevchenko, E. V., Talapin, D. V., O'Brien, S. \& Murray, C. B. Polymorphism in $A B_{13}$ nanoparticle superlattices: An example of semiconductor-metal metamaterials. J. Am. Chem. Soc. 127, 8741-8747 (2005)

8. Murray, M. J. \& Sanders, J. V. Close-packed structures of spheres of two different sizes II. The packing densities of likely arrangements. Phil. Mag. A 42, 721-740 (1980)

9. Eldridge, M. D., Madden, P. A. \& Frenkel, D. Entropy-driven formation of a superlattice in a hard-sphere binary mixture. Nature 365, 35-37 (1993)

10. Leunissen, M. E. et al. lonic colloidal crystals of oppositely charged particles. Nature 437, 235-240 (2005).

11. Bartlett, P. \& Campbell, A. I. Three-dimensional binary superlattices of oppositely charged colloids. Phys. Rev. Lett. 95, 128302 (2005).

12. Bolhuis, P. G., Frenkel, D., Mau, S.-C. \& Huse, D. A. Entropy difference between the face-centred cubic and hexagonal close-packed crystal structures. Nature 388, 235-236 (1997).

13. Pusey, P. N. \& van Megen, W. Phase behaviour of concentrated suspensions of nearly hard colloidal spheres. Nature 320, 340-342 (1986).

14. Wong, S., Kitaev, V. \& Ozin, G. A. Colloidal crystal films: Advances in universality and perfection. J. Am. Chem. Soc. 125, 15589-15598 (2003).

15. Cottin, X. \& Monson, P. A. Substitutionally ordered solid solutions of hard spheres. J. Chem. Phys. 102, 3354-3360 (1995).

16. Sanders, J. V. \& Murray, M. J. Ordered arrangements of spheres of two different sizes in opal. Nature 275, 201-203 (1978)

17. Hachisu, S. \& Yoshimura, S. Optical demonstration of crystalline superstructures in binary mixtures of latex globules. Nature 283, 188-189 (1980).

18. Shim, M. \& Guyot-Sionnest, P. Permanent dipole moment and charges in colloidal semiconductor quantum dots. J. Chem. Phys. 111, 6955-6964 (1999).

19. Krauss, T. D. \& Brus, L. E. Charge, polarizability, and photoionization of single semiconductor nanocrystals. Phys. Rev. Lett. 83, 4840-4843 (1999).

20. Islam, M. A. \& Herman, I. P. Electrodeposition of patterned CdSe nanocrystal films using thermally charged nanocrystals. Appl. Phys. Lett. 80, 3823-3825 (2002).

21. O'Brien, R. W. \& White, L. R. Electrophoretic mobility of a spherical colloidal particle. J. Chem. Soc. Farad. Trans. II 74, 1607-1626 (1978).
22. Yin, Y. \& Alivisatos, A. P. Colloidal nanocrystal synthesis and the organic-inorganic interface. Nature 437, 664-670 (2005).

23. Korgel, B. A., Fullam, S., Connolly, S. \& Fitzmaurice, D. Assembly and self-organization of silver nanocrystal superlattices: Ordered "soft spheres". J. Phys. Chem. B 102, 8379-8388 (1998).

24. Cho, K.-S., Talapin, D. V., Gaschler, W. \& Murray, C. B. Designing PbSe nanowires and nanorings through oriented attachment of nanoparticles. J. Am. Chem. Soc. 127, 7140-7147 (2005).

25. Ohara, P. C. Leff, D. V. Heath, J. R. \& Gelbart, W. M. Crystallization of opals from polydisperse nanoparticles. Phys. Rev. Lett. 75, 3466-3469 (1995).

26. Rabani, E., Reichman, D. R., Geissler, P. L. \& Brus, L. E. Drying-mediated self-assembly of nanoparticles. Nature 426, 271-274 (2003).

27. Prasad, B. L. V., Stoeva, S. I., Sorensen, C. M. \& Klabunde, K. J. Digestive ripening of thiolated gold nanoparticles: The effect of alkyl chain length. Langmuir 18, 7515-7520 (2002).

28. Hyeon, T., Lee, S. S., Park, J., Chung, Y. \& Na, H. B. Synthesis of highly crystalline and monodisperse maghemite nanocrystallites without a size-selection process. J. Am. Chem. Soc. 123, 12798-12801 (2001).

29. Hines, M. A. \& Scholes, G. D. Colloidal PbS nanocrystals with size-tunable near-infrared emission: Observation of post-synthesis self-narrowing of the particle size distribution. Adv. Mater. 15, 1844-1849 (2003).

30. Zhang, Y.-W., Sun, X., Si, R., You, L.-P. \& Yan, C.-H. Single-crystalline and monodisperse $\mathrm{LaF}_{3}$ triangular nanoplates from a single-source precursor. J. Am. Chem. Soc. 127, 3260-3261 (2005).

Supplementary Information is linked to the online version of the paper at www.nature.com/nature.

Acknowledgements We thank V. Perebeinos, A. van Blaaderen, V. Crespi, I. Herman and L. E. Brus for discussions and R. L. Sandstrom for technical support. This work was partially supported by the MRSEC Program of the National Science Foundation, and by the New York State Office of Science, Technology and Academic Research (NYSTAR). S.O. is grateful for support from the DOE and an NSF CAREER award.

Author Contributions E.V.S. and D.V.T. contributed equally to this work. E.V.S. and D.V.T. carried out syntheses of nanoparticles, and E.V.S. investigated formation of binary nanoparticle superlattices. E.V.S. and D.V.T. performed modelling and structural assignment of self-assembled binary superlattices. E.V.S., D.V.T. and N.A.K. studied electrophoretic mobility of nanoparticles and worked on modelling self-assembly phenomena in binary nanoparticle colloids. S.O. and C.B.M. initiated and supervised the work. D.V.T. and C.B.M. wrote the paper. All authors discussed the results and commented on the manuscript.

Author Information Reprints and permissions information is available at npg.nature.com/reprintsandpermissions. The authors declare no competing financial interests. Correspondence and requests for materials should be addressed to D.V.T. (dvtalapin@lbl.gov) or C.B.M. (cbmurray@us.ibm.com). 